\title{
Coeliac disease: Oral ulcer prevalence, assessment of risk and association with gluten-free diet in children
}

\author{
G. Campisi ${ }^{\text {a }}$, C. Di Liberto ${ }^{\mathrm{a}}$, A. Carroccio ${ }^{\mathrm{b}}$, D. Compilato ${ }^{\mathrm{a}}$, \\ G. Iacono ${ }^{\text {c }}$, M. Procaccini ${ }^{\mathrm{d}}$, G. Di Fede ${ }^{\mathrm{b}}$, L. Lo Muzio $^{\mathrm{e}}$, \\ A. Craxi ${ }^{f}$, C. Catassi ${ }^{g}$, C. Scully ${ }^{\mathrm{h}, *}$ \\ ${ }^{a}$ Department of Oral Sciences, University Hospital of Palermo, Palermo, Italy \\ ${ }^{\mathrm{b}}$ Department of Gastroenterology, University Hospital of Palermo, Palermo, Italy \\ ${ }^{c}$ Pediatric Gastroenterology, Di Cristina Hospital of Palermo, Palermo, Italy \\ ${ }^{\mathrm{d}}$ Institute of Dental Science, Marche Polytechnic University, Ancona, Italy \\ ${ }^{\mathrm{e}}$ Department of Surgical Sciences, University of Foggia, Italy \\ ${ }^{\mathrm{f}}$ Department of Internal Medicine, University Hospital of Palermo, Palermo, Italy \\ g Department of Pediatrics, Marche Polytechnic University, Ancona, Italy \\ ${ }^{\mathrm{h}}$ Eastman Dental Institute, University College London, 256 Gray's Inn Road, London WC1X 8LD, UK
}

Received 3 January 2007; accepted 16 October 2007

Available online 11 December 2007

\begin{abstract}
Aims. Oral mucosal lesions may be markers of chronic gastrointestinal disorders, such as those causing malabsorption. Our objectives were to assess the prevalence of recurrent oral aphthous-like ulcers in coeliac disease patients living in the Mediterranean area, and to evaluate the impact of a gluten-free diet.

Methods. A test group of 269 patients (age range 3-17 years) with coeliac disease confirmed both serologically and histologically was compared with a control group of 575 otherwise clinically healthy subjects for the presence, or a positive history of aphthous-like ulcers. Coeliac disease patients with aphthous-like ulcers were re-evaluated 1-year after starting a gluten-free diet.

Results. Aphthous-like ulcers were found significantly more frequently in coeliac disease, in $22.7 \%$ (61/269) of patients with coeliac disease versus $7.1 \%(41 / 575)$ of controls $(p=<0.0001$; chi-square $=41.687$; odds ratio $=4.3123 ; 95 \%$ confidence interval $=2.7664: 6.722$ ). Most coeliac disease patients with aphthous-like ulcers and adhering strictly to gluten-free diet $(71.7 \% ; 33 / 46)$ reported significant improvement on gluten-free diet, with no or reduced episodes of aphthous-like ulcers $(p=0.0003$; chi-square $=13.101 ;$ odds ratio $=24.67 ; 95 \%$ confidence interval $=2.63: 231.441$ ).

Conclusions. The epidemiological association found between coeliac disease and aphthous-like ulcers suggests that recurrent aphthous-like ulcers should be considered a risk indicator for coeliac disease, and that gluten-free diet leads to ulcer amelioration.
\end{abstract}

(C) 2007 Editrice Gastroenterologica Italiana S.r.l. Published by Elsevier Ltd. All rights reserved.

Keywords: Aphthous ulcer; Coeliac disease; Gluten free-diet

\section{Introduction}

Coeliac disease (CD) can manifest with a "typical" enteropathy, characterized by a malabsorption syndrome, but this is far less common than the atypical and/or silent

\footnotetext{
* Corresponding author. Tel.: +44 207915 1038; fax: +44 2079151039.

E-mail address: Scully.C@eastman.ucl.ac.uk (C. Scully).
}

forms which are clinically heterogeneous, and therefore often unsuspected [1].

Because of the wide range of clinical manifestations patients could be referred to any specialist [2]. Attention should also be paid to patients with some oral lesions, such as recurrent aphthous stomatitis (RAS) [3,4], symmetric and systematic dental enamel defects [5-8], and non-specific atrophic glossitis [9]. RAS is one of the most common mucosal diseases and is characterized by "multiple recurrent 
painful, round or ovoid ulcers with circumscribed margins, erythematous haloes, and yellow or grey floors, appearing first in childhood or adolescence" [10,11]. Scully [12] suggested that the term "recurrent aphthous stomatitis" should be reserved for those ulcers in patients without systemic diseases, whereas those with a similar clinical appearance, but found in systemic and intestinal (i.e. CD and inflammatory bowel diseases) $[13,14]$ disorders, should be properly termed "aphthous-like ulcers' (ALU). The reported prevalence of mouth ulcers in $\mathrm{CD}$ patients ranges widely from $3.7 \%$ to $33.3 \%[7,9,15,16]$. Furthermore, the response of ALU patients to a gluten-free diet (GFD) is controversial: while some authors report their amelioration or resolution after GFD [15,17-19] one double-blind placebo-controlled trial discounted this [20].

We therefore performed a case-control study in which the primary objectives were to assess the prevalence of ALU in a large population of $\mathrm{CD}$ patients living in the Mediterranean area, and to assess the impact of a GFD on the ALU.

\section{Material and methods}

The study population consisted of 844 subjects living in the Mediterranean area. Local ethical committee approval was obtained.

The test group consisted of $269 \mathrm{CD}$ patients ( 163 females, 106 males; range 3-17 years) consecutively enrolled between 2004 and 2006 in two Italian centres: the Universities of Palermo and Ancona. CD diagnosis was based both on positive serology (IgA and/or IgG antibodies to human tissue transglutaminase [Ab-htTG]), together with positive histological evidence of villous atrophy with crypt hyperplasia and increase in intraepithelial lymphocytes, and the disappearance of symptoms and normalization of serum Ab-htTG after GFD.

The control group was composed of 575 otherwise healthy subjects' age/gender-matched (343 females, 232 males; range 3-17 years) and living in the same geographical area as the $\mathrm{CD}$ group. Controls were recruited (by simple randomization) at a day nursery, and at Primary and Secondary Schools during a health prevention programme for oral diseases. These subjects were healthy, with no disease or previous positive medical history, or family history of CD and showed normal growth (weight/height ratio between 25 th and 75 th centiles).

Immediately after $\mathrm{CD}$ diagnosis, the patients in the test group underwent an intra-oral examination. Test and control patients were examined after obtaining informed consent in the presence of their parents, who were asked whether there was a clinical history of ALU. In this study we included both ALU observed clinically and ulcers noted by parents or patients, or reported in hospital records.

According to Stanley [21] RAS has three main clinical presentations: minor, major or herpetiform ulcerations. The first are round ulcers less than $10 \mathrm{~mm}$ in diameter, while the major are clinically similar to the minor but are larger than $10 \mathrm{~mm}$ in diameter and more persistent [11,22].

All patients were examined in conventional dental chairs, using a dental operating light. All evaluations were performed independently by two of the authors trained in oral health survey (DC and MP) and tested for concordance.

CD patients with ulcers or a history of ALU at the first visit were advised a GFD and re-evaluated one year after beginning this diet. At the second visit, adherence to GFD, serum anti-htTG and the frequency and severity of ALU were recorded.

Finally, all the controls positive for ALU were tested for serum coeliac antibodies to exclude CD.

\section{Statistical analyses}

Data were analysed by means of StatView for Windows (SAS Inc. v. 5.0.1, Cary, NC, USA). To measure the association level, odds ratio (OR) and the $95 \%$ corresponding test-based confidence interval (CI) were calculated. Student's $t$-test was used to calculate significant differences between test patients and controls at baseline for ordinal variables. Chi-square test was used to assess statistical differences between categorical variables. In all evaluations, $p$-values $<0.05$ were considered statistically significant. The concordance rate of the oral evaluation between the observers was evaluated using Cohen's kappa statistic, as measuring agreement beyond that expected by chance (expressed as a coefficient ranging from 0 to 1.00) [23].

\section{Results}

No significant differences in age and gender $(p>0.2$ by Student's $t$-test and chi-square test, respectively) were detected between the test and control groups.

There was a substantial agreement $(\kappa=0.87)$ between the observers for ALU assessment.

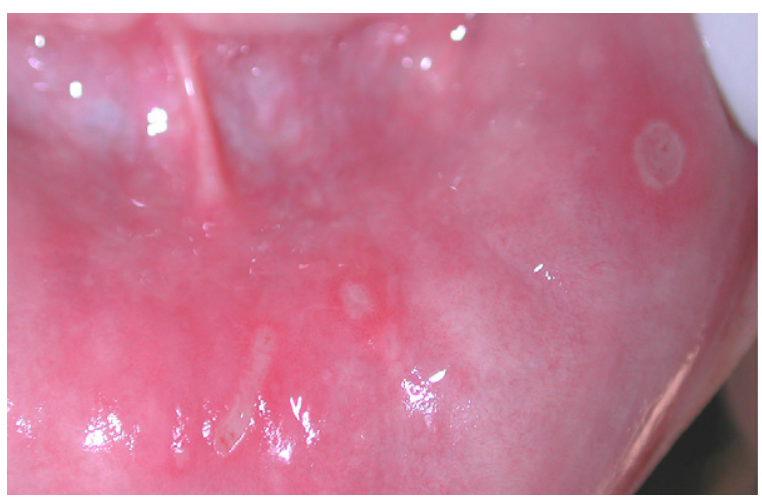

Fig. 1. ALU minor in patient with untreated CD. 
ALU was found in 61/269 (22.7\%) of CD patients versus $41 / 575(7.1 \%)$ of the control group, showing a highly significant association of ALU with CD $(p=<0.0001$; chisquare $=41.687 ; \mathrm{OR}=4.3123 ; 95 \% \mathrm{CI}=2.7664-6.722)$. The majority of ALU in both groups were of the minor type $(90.1 \%$ versus $87.8 \% ; p>0.2)$ (Fig. 1). There were no differences in the number of episodes, rate of occurrence and duration $(p>0.2)$.

None of these forty-one control patients with ALU had positive serological markers for $\mathrm{CD}$.

At the 1-year visit, there were 8 drop-outs (13.1\%), therefore the remaining $53 \mathrm{CD}$ patients with ALU were interviewed and examined. Forty-six of these 53 patients adhered strictly to GFD (87\%): 33 (71.7\%) had had no ALU, while in $4(8.7 \%)$ ulcers were reduced in frequency and severity and in $9(19.6 \%)$ there was no change in ALU history. In the other 7/53 patients who failed to strictly adhere to the GFD, six did not report any improvement in ALU, and Ab-htTG remained elevated. The difference in ALU history between test patients adhering versus those not adhering to GFD was significant $(p=0.0003$; chi-square $=13.101 ; \mathrm{OR}=24.67$; $95 \% \mathrm{CI}=2.63: 231.441)$.

\section{Discussion}

Atypical CD is characterized by non-specific intestinal complaints or by extra-intestinal manifestations including dental enamel defects, or mouth ulcers.

The frequency of true RAS is debated, in fact, the literature reports different frequencies of RAS in the general young population: in some Brazilian studies it has been found in $0.9-1.57 \%$ [24,25]; Crivelli et al. [26] described it in 10.87\% of Argentinian children, and Kleinman observed RAS only in $1.23 \%$ of 39206 United States schoolchildren [27,28]. ALU, also usually labelled RAS, have been reported in CD but with controversial frequencies $[4,7,29]$. In the present study, the prevalence of ALU was $22.7 \%$ in $269 \mathrm{CD}$ patients versus $7.1 \%$ in 575 individuals from the general population living in the same area, with an odds ratio of suffering from ALU in the risk population of about 4.3 , whilst other studies have shown a prevalence of 3.7-41.0\% (Table 1). These differences may be explained by environmental, dietetic and, above all, genetic factors.

Thus, our data support the hypothesis of a link between ALU and CD, but others have reported that only $5 \%$ of outpatients who initially present recurrent ulcers have evidence of CD [17,30]. Consequently, in the absence of other systemic signs, screening for gluten-sensitive enteropathy in individuals with RAS has generally been considered fruitless [4]. However, on the basis of our finding of a very high percentage ALU in CD patients in a large population, we suggest that ALU should be considered, as proposed by Sedghizadeh [4], a "risk indicator" for CD rather than a "risk factor." Hence, the presence of oral aphthae should not be underestimated, but should be considered a potential marker of underly-
Table 1

Prevalence of ALU in CD Patients

\begin{tabular}{|c|c|c|c|}
\hline Authors & $n, \mathrm{CD}$ patients & Prevalence & $p$ value \\
\hline Sedghizadeh et al. [4] & 61 & 41.0 & N.S. ${ }^{*}$ \\
\hline Procaccini et al. [3] & 50 & 36.0 & Significant \\
\hline Bucci et al. [7] & 72 & 33.3 & N.S. \\
\hline Andersson-Wenckert et al. [31] & 19 & 26.3 & N.S. \\
\hline Present study & 269 & 22.7 & Significant \\
\hline Sood et al. [29] & 96 & 19.8 & Significant \\
\hline Petrecca et al. [6] & 29 & 17.0 & Not calculated \\
\hline Majorana et al. [15] & 113 & 16.8 & Not calculated \\
\hline Lähteenoja et al. [9] & 128 & 3.7 & Not calculated \\
\hline
\end{tabular}

ing disease, above all if a patient has a positive personal and/or family clinical history. Consequently, serum CD markers (anti-endomysial and anti-transglutaminase antibodies) should also be assayed in oligosymptomatic or asymptomatic subjects showing ALU. However, it must be remembered that recurrent aphthous stomatitis can also be associated with Behçet's disease and other inflammatory bowel diseases [13] and consequently the association between ALU and CD cannot be considered specific. Further studies will determine the positive predictive value of ALU presence in CD diagnosis.

A final point worthy of note is that in the CD patients suffering from ALU in our study who strictly adhered to GFD there was a significant improvement in ALU history 1 year after commencement of GFD, whereas no improvement was observed in the $\mathrm{CD}$ patients who did not adhere to GFD.

\section{Practice points}

- Among the atypical clinical presentations of $C D$ several oral manifestations have been described (e.g. dental enamel defects, recurrent mouth ulcers) and these signs could help to identify CD cases.

- Conflicting data have been published on the real frequency of recurrent aphthous stomatitis (RAS) in CD and there are few data on the effect of a gluten-free diet on RAS in CD patients.

- We found a higher frequency of $23 \%$ of mouth ulcers in a large group of CD patients compared to controls $(O R=4.3123)$ and showed that these lesions disappeared or significantly improved only in CD patients who adhered to a gluten-free diet. 


\section{Research agenda}

- Studies to evaluate the positive and negative predictive value of mouth ulcers presence in CD diagnosis are needed.

- The pathogenesis of mouth ulcers in CD should be evaluated in future studies taking into account both immunological and nutritional parameters.

- Other oral signs and manifestations should be considered to evaluate whether the presence of oral lesions in general could improve diagnostic accuracy in CD diagnosis.

\section{Conflict of interest statement}

None declared.

\section{References}

[1] Catassi C, Fasano A. New developments in childhood celiac disease. Curr Gastroenterol Rep 2002;4:238-43.

[2] Green PH, Jabri B. Coeliac disease. Lancet 2003;362:383-91.

[3] Procaccini M, Campisi G, Bufo P, Compilato D, Massaccesi C, Catassi $\mathrm{C}$, et al. Lack of association between celiac disease and dental enamel hypoplasia in a case-control study from an Italian central region. Head Face Med 2007;3:25.

[4] Sedghizadeh PP, Shuler CF, Allen CM, Beck FM, Kalmar JR. Celiac disease and recurrent aphthous stomatitis: a report and review of the literature. Oral Surg Oral Med Oral Pathol Oral Radiol Endod 2002;94:474-8.

[5] Aine L, Maki M, Collin P, Keyrilainen O. Dental enamel defects in celiac disease. J Oral Pathol Med 1990;19:241-5.

[6] Petrecca S, Giammaria G, Giammaria AF. Oral cavity changes in the child with celiac disease. Minerva Stomatol 1994;43:137-40.

[7] Bucci P, Carile F, Sangianantoni A, D’Angiò F, Santarelli A, Lo Muzio L. Oral aphthous ulcers and dental enamel defects in children with coeliac disease. Acta Paediatr 2006;95:203-7.

[8] Wierink CD, van Diermen DE, Aartman IH, Heymans HS. Dental enamel defects in children with coeliac disease. Int J Paediatr Dent 2007;17:163-8.

[9] Lähteenoja H, Toivanen A, Viander M, Mäki M, Irjala K, Räihä I, et al. Oral mucosal changes in coeliac patients on a gluten-free diet. Eur J Oral Sci 1998;106:899-906.

[10] Scully C, Felix DH. Oral medicine-update for the dental practitioner Aphthous and other common ulcers. Br Dent J 2005;199:259-64.

[11] Scully C, Porter S. Oral mucosal disease: Recurrent aphthous stomatitis. Br J Oral Maxillofac Surg 2007 Sep. 10 [Epub ahead of print].
[12] Scully C. Clinical practice. Aphthous ulceration. N Engl J Med 2006;355:165-72.

[13] Field EA, Allan RB. Review article: oral ulcerationaetiopathogenesis, clinical diagnosis and management in the gastrointestinal clinic. Alim Pharmacol Ther 2003;18:949-62.

[14] Snyder MB, Cawson RA. Oral changes in Crohn's disease. J Oral Surg 1976;34:594-9.

[15] Majorana A, Sapelli PL, Malagoli A, Meini A, Pillan MN, Duse M, et al. Celiac disease and recurrent aphthous stomatitis. The clinical and immunogenetic aspects. Minerva Stomatol 1992;41:33-40.

[16] Meini A, Pillan MN, Plebani A, Ugazio AG, Majorana A, Sapelli PL. High prevalence of DRW10 and DQW1 antigens in celiac disease associated with recurrent aphthous stomatitis. Am J Gastroenterol 1993;88:972.

[17] Wray D. Gluten-sensitive recurrent aphthous stomatitis. Dig Dis Sci 1981;26:737-40.

[18] Biel K, Böhm M, Luger TA, Bonsmann G. Long-standing oral aphthae - a clue to the diagnosis of coeliac disease. Dermatology 2000;200:340.

[19] Scully C, Gorsky M, Lozada-Nur F. The diagnosis and management of recurrent aphthous stomatitis: a consensus approach. J Am Dent Assoc 2003;134:200-7.

[20] Hunter IP, Ferguson MM, Scully C, Galloway AR, Main AN, Russell RI. Effects of dietary gluten elimination in patients with recurrent minor aphthous stomatitis and no detectable gluten enteropathy. Oral Surg Oral Med Oral Pathol 1993;75:595-8.

[21] Stanley HR. Aphthous lesions. Oral Surg Oral Med Oral Pathol 1972;33:407-16.

[22] Natah SS, Konttinen YT, Enattah NS, Ashammakhi N, Sharkey KA, Häyrinen-Immonen R. Recurrent aphthous ulcers today: a review of the growing knowledge. Int J Oral Maxillofac Surg 2004;33:221-34.

[23] Cohen J. Weighted kappa: nominal scale agreement with provision for scaled disagreement or partial credit. Psychol Bull 1968;70:213-20.

[24] dos Santos PJ, Bessa CF, de Aguiar MC, do Carmo MA. Cross-sectional study of oral mucosal conditions among a central Amazonian Indian community, Brazil. J Oral Pathol Med 2004;33:7-12.

[25] Bessa CF, Santos PJ, Aguiar MC, do Carmo MA. Prevalence of oral mucosal alterations in children from 0 to 12 years old. J Oral Pathol Med 2004;33:17-22.

[26] Crivelli MR, Aguas S, Adler I, Quarracino C, Bazerque P. Influence of socioeconomic status on oral mucosa lesion prevalence in schoolchildren. Commun Dent Oral Epidemiol 1988;16:58-60.

[27] Rioboo-Crespo Mdel R, Planells-del Pozo P, Rioboo-Garcia R. Epidemiology of the most common oral mucosal diseases in children. Med Oral Pathol Oral Cir Bucal 2005;10:376-87.

[28] Kleinman DV, Swango PA, Pindborg JJ. Epidemiology of oral mucosal lesions in United States schoolchildren: 1986-87. Commun Dent Oral Epidemiol 1994;22:243-53.

[29] Sood A, Midha V, Sood N, Malhotra V. Adult celiac disease in northern India. Indian J Gastroenterol 2003;22:124-6.

[30] Ferguson MM, Wray D, Carmichael HA, Russell RI, Lee FD. Coeliac disease associated with recurrent aphthae. Gut 1980;21:223-6.

[31] Andersson-Wenckert I, Blomquist HK, Fredrikzon B. Oral health in coeliac disease and cow's milk protein intolerance. Swed Dent J 1984;8:9-14. 